\title{
On Null Curves in Minkowski 3-Space and Its Fractal Folding
}

\author{
A.E. El-Ahmady ${ }^{1}$, Malak E. Raslan ${ }^{2}$ \& A.T. M-Zidan ${ }^{1}$ \\ ${ }^{1}$ Department of Mathematics, Faculty of Science, Tanta University, Egypt \\ ${ }^{2}$ Department of Mathematics, Faculty of Science, Damietta University, Egypt \\ Correspondence: A.T. M-Zidan, Mathematics Department, Faculty of Science, Damietta University, Egypt. \\ E-mail:atm_zidan@yahoo.com
}

Received: February 21, 2019

Accepted: March 26, 2020

Online Published: March 27, 2020

doi:10.5539/mas.v14n4p90

URL: https://doi.org/10.5539/mas.v14n4p90

\begin{abstract}
In this paper, a form for Frenet equations of all null curves in Minkowski 3-space has been presented. New types of foldings of curves are obtained. The connection between folding, deformation and Frenet equations of curves are also deduced.
\end{abstract}

Keywords: Minkowski 3-space, null curves, conditional fractal folding, deformation, Frenet equations

AMS (2010): 53A35, 51B20, 58C05.

\section{Introduction}

The Minkowski 3-space $E_{1}^{3}$ is the Euclidean 3-space $E^{3}$ provided with the standard flat metric given by

$$
g=d x_{1}^{2}+d x_{2}^{2}-d x_{3}^{2}
$$

where $\left(x_{1}, x_{2}, x_{3}\right)$ is a rectangular coordinate system in $E_{1}^{3}$. Since $g$ is an indefinite metric, recall that a vector $v \in E_{1}^{3}$ is said space-like if $g(v, v)>0$ or $v=0$, time-like if $g(v, v)<0$ and null (light-like) if $g(v, v)=0$ and $v \neq 0$. Similarly, an arbitrary curve $\alpha=\alpha(s)$ in $E_{1}^{3}$ can locally be space-like, time- like or null(light-like), if all of its velocity vectors $\alpha^{\prime}(s)$ are respectively, space-like, time-like or null (light-like) respectively. Space-like or time-like curve $\alpha(s)$ is said to be parameterized by arc length function s, if $g\left(\alpha^{\prime}(s), \alpha^{\prime}(s)\right)= \pm 1$. The velocity of the curve $\alpha(s)$ is given by $\left\|\alpha^{\prime}(s)\right\|$. A curve $\alpha$ is said to be regular if $\alpha^{\prime}(t) \neq 0$ for all $t \in I, \alpha \in L^{n}$ is space-like if its velocity vectors $\alpha^{\prime}$ are space-like for all $t \in I$, similarly for time-like and null. If $\alpha$ is a null curve, we can re-parameterize it such that, $\left\langle\alpha^{\prime}(t), \alpha^{\prime}(t)\right\rangle=0$ and $\alpha^{\prime}(t) \neq 0$, recall the norm of a vector $v$ is given by $\|v\|=\sqrt{|g(v, v)|}$.

Given a unit speed curve $\alpha(s)$ in Minkowski space $E_{1}^{3}$ we can possible define a Frenet frame $\{T(s), N(s), B(s)\}$ associated for each point $s$. Where $T(s), N(s)$ and $B(s)$ are the tangent, normal and binormal vector field (A. E. El-Ahmady \& A.T.M. Zidan. 2019) (A. E. El-Ahmady \& E. Al-Hesiny. 2013) (R. Lopez. 2008) (R. Aslaner, A. Ihsan Boran. 2009).

\section{Preliminary Notes}

Let $\alpha(\mathrm{s})$ be a curve in $E_{1}^{3}$. Then for the unit speed curve $\alpha(s)$ with non-null frame vectors, we distinguish three cases depending on the causal character of $T(s)$ and its Frenet equations are as follows,

$$
\left(\begin{array}{l}
T^{\prime} \\
N^{\prime} \\
B^{\prime}
\end{array}\right)=\left(\begin{array}{ccc}
0 & k & 0 \\
\mu_{1} k & 0 & \mu_{2} \tau \\
0 & \mu_{3} \tau & 0
\end{array}\right)\left(\begin{array}{l}
T \\
N \\
B
\end{array}\right) .
$$

We write the following subcases,

Case 1. If $\alpha(s)$ is time-like curve in $E_{1}^{3}$, then $T$ is time-like vector and $T^{\prime}$ is space-like vector. Then $\mu_{i}(1<i<3)$, read $\mu_{1}=\mu_{2}=1, \mu_{3}=-1, T, B$ and $N$ are mutually orthogonal vectors satisfying the equations, $g(N, N)=g(B, B)=1, g(T, T)=-1$.

Case 2. If $\alpha(s)$ is space like curve in $\mathrm{E}_{1}^{3}$, then $T$ is space like vector, since $T^{\prime}(s)$ is orthogonal to the space like vector $T(s), T^{\prime}(s)$ may be space like, time-like or light like. Thus we distinguish three cases according to $T^{\prime}(s)$. 
Case 2.1. If the vector $T^{\prime}(s)$ is space-like, $N$ is space like vector and $B$ is time-like vector. Then $\mu_{i}(1<i<$ 3) read $\mu_{1}=-1, \mu_{2}=\mu_{3}=1, T, N$ and Bare mutually orthogonal vectors satisfying $g(T, T)=$ $g(N, N)=1, g(B, B)=-1$.

Case 2.2. If the vector $T^{\prime}(s)$ is time-like, $N$ is time-like vector and $B$ is space-like vector. Then $\mu_{i}(1<i<$ 3) read $\mu_{1}=\mu_{2}=\mu_{3}=1$, where the orthogonal vectors $T, N$ and $B$ are satisfying $g(T, T)=g(B, B)=$ $1, g(N, N)=-1$.

Case 2.3. If the vector $T^{\prime}(s)$ is light like for all $s, N(s)=T^{\prime}(s)$ is light like vector and $B(s)$ is unique light like vector such that $g(N, B)=-1$ and it is orthogonal to $T$. The Frenet equations are

$$
\left(\begin{array}{l}
T^{\prime} \\
N^{\prime} \\
B^{\prime}
\end{array}\right)=\left(\begin{array}{ccc}
0 & 1 & 0 \\
0 & \tau & 0 \\
1 & 0 & -\tau
\end{array}\right)\left(\begin{array}{l}
T \\
N \\
B
\end{array}\right) .
$$

Case 3. If $\alpha(s)$ is light like curve in $\mathrm{E}_{1}^{3}, g(N, N)>0$, when the parameterization is pseudo- arc so $g(N, N)=1$ with $g(T, T)=0, g(B, B)=0, g(T, N)=0$, and $B(s)$ is unique light like vector such that $g(T, B)=-1$ and it is orthogonal to $N$ the pseudo torsion of $\alpha(s)$ be $\tau=-\left\langle N^{\prime}, B\right\rangle$, then the Frenet equations of $\alpha(s)$ are

$$
\left(\begin{array}{l}
T^{\prime} \\
N^{\prime} \\
B^{\prime}
\end{array}\right)=\left(\begin{array}{lll}
0 & k & 0 \\
\tau & 0 & k \\
0 & \tau & 0
\end{array}\right) *
$$

Where the curvature $k$ can take only two values, 0 when $\alpha$ is a straight null line, or 1 in all other cases (J. Walrave. 1995).

A regular curve $\alpha: I \rightarrow E_{1}^{3}$ is called a null curve if $\alpha^{\prime}$ is light like, that is $\left\langle\alpha^{\prime}, \alpha^{\prime}\right\rangle=0$ (M. P. Docarmo. 1992).

Let $M$ and $N$ be two smooth manifolds of dimensions $m$ and $n$ respectively. A map $f: M \rightarrow N$ is said to be an isometric folding of $M$ into $N$ if and only if for every piecewise geodesic path $\gamma: I \rightarrow M$ the induced path $f$ o $\gamma: I \rightarrow N$ is piecewise geodesic and of the same length as $\gamma$, if $f$ does not preserve the length it is called topological folding (A. E. El-Ahmady. 2007) (A. E. El-Ahmady \& E. Al-Hesiny. 2013).

A map $d: M \rightarrow M^{*}$ such that $M^{*}=d(M)$ where $M$ and $M^{*}$ are two smooth Riemannian manifolds is called deformation map if $d$ is differentiable and has differentiable inverse. A deformation map $d: M \rightarrow M^{*}$ where $M$ and $M^{*}$ are two smooth Riemannian manifolds is called regular deformation if $\left.\forall x, y \in M, K(x)=K(y)\right) \Leftrightarrow$ $K(d(x))=K(d(y)), K(x)$ is the curvature at the point $x \in M$, when $(x)=K(d(x)) \forall x \in M$, it is the identity deformation which is regular deformation (M. P. Docarmo. 1992).

Definition 2.1. Let $\mathrm{u}=\left(\mathrm{u}_{1}, \mathrm{u}_{2}, \mathrm{u}_{3}\right)$ and $\mathrm{v}=\left(\mathrm{v}_{1}, \mathrm{v}_{2}, \mathrm{v}_{3},\right)$ be vectors in $\mathrm{E}_{1}^{3}$, the vector product in Minkowski space-time $\mathrm{E}_{1}^{3}$ is defined by the determinant

$$
\mathrm{u} \wedge \mathrm{v}=\left|\begin{array}{ccc}
e_{1} & e_{2} & -e_{3} \\
\mathrm{u}_{1} & \mathrm{u}_{2} & \mathrm{u}_{3} \\
\mathrm{v}_{1} & \mathrm{v}_{2} & \mathrm{v}_{3}
\end{array}\right| .
$$

Where $e_{1}, e_{2}$ and $e_{3}$ are mutually orthogonal vectors (coordinate direction vectors).

\section{Form of Frenet Equations of Null Curves in Minkowski 3-Space}

Theorem 3.1. Let $\xi(s)$ be a null curve in $\mathrm{E}_{1}^{3}$ with the standard flat metric given by $g=d x_{1}^{2}+d x_{2}^{2}-d x_{3}^{2}$. Then the bi-normal vector of $\xi(s)$ can be calculated by the form,

$B(s)=\left(\frac{-1}{\Delta_{1,2}}\left(\Delta_{2,3} b_{3}+x_{2}^{\prime \prime}\right), \frac{1}{\Delta_{1,2}}\left(\Delta_{1,3} b_{3}+x_{1}^{\prime \prime}\right), \frac{-\left(1+x_{3}^{\prime \prime 2}\right)}{2 x_{3}^{\prime}}\right), \Delta_{1,2} \neq 0, x_{3}^{\prime} \neq 0$.

Where $\Delta_{2,3}=\left(x_{2}^{\prime} x_{3}^{\prime \prime}-x_{3}^{\prime} x_{2}^{\prime \prime}\right), \Delta_{1,3}=\left(x_{1}^{\prime} x_{3}^{\prime \prime}-x_{3}^{\prime} x_{1}^{\prime \prime}\right)$ and $\Delta_{1,2}=\left(x_{1}^{\prime} x_{2}^{\prime \prime}-x_{2}^{\prime} x_{1}^{\prime \prime}\right)$.

Proof. Let $\xi(s)=\left(x_{1}(s), x_{2}(s), x_{3}(s)\right)$, be the parametric equation of any null curve in $E_{1}^{3}$ where the tangent vector $T(s)=\left(x_{1}^{\prime}(s), x_{2}^{\prime}(s), x_{3}^{\prime}(s)\right)$ and the normal vector $N(s)=T^{\prime}(s)=\left(x_{1}^{\prime \prime}(s), x_{2}^{\prime \prime}(s), x_{3}^{\prime \prime}(s)\right)$. To calculate the bi-normal vector of the curve $\xi(s)$, let $B(s)=\left(b_{1}, b_{2}, b_{3}\right)$,

since $B(s)$ is unique light like vector, hence

$\langle B, B\rangle=0$ and so,

$$
b_{1}{ }^{2}+{b_{2}}^{2}-b_{3}{ }^{2}=0 \text {. }
$$

Also, $g(T, B)=-1$ and so, 
$x_{1}^{\prime} b_{1}+x_{2}^{\prime} b_{2}-x_{3}^{\prime} b_{3}=-1$. (2)

Since $B$ is orthogonal to $N$ where $\langle N, B\rangle=0$ so we get,

$x_{1}^{\prime \prime} b_{1}+x_{2}^{\prime \prime} b_{2}-x_{3}^{\prime \prime} b_{3}=0$. (3)

Multiply equation (2) by $x_{1}^{\prime \prime}$ and equation (3) by $x_{1}^{\prime}$ and subtracting the product equations so we get,

$b_{2}=\frac{1}{\Delta_{1,2}}\left(\Delta_{1,3} b_{3}+x_{1}^{\prime \prime}\right), \Delta_{1,2} \neq 0$. (4)

Multiply equation 2 by $x 2^{\prime \prime}$ and equation 3 by $x 2^{\prime}$ and subtracting the product equations. Then,

$b_{1}=\frac{-1}{\Delta_{1,2}}\left(\Delta_{2,3} b_{3}+x_{2}^{\prime \prime}\right), \Delta_{1,2} \neq 0$.

By substituting equations 4 and 5 in equation 1 . Then,

$\left(\Delta_{2,3}{ }^{2}+\Delta_{1,3}{ }^{2}-\Delta_{1,2}{ }^{2}\right) b_{3}{ }^{2}+x_{1}^{\prime \prime 2}+x_{2}^{\prime \prime 2}+2\left(\Delta_{1,3} x_{1}^{\prime \prime}+\Delta_{2,3} x_{2}^{\prime \prime}\right) b_{3}=0$.

But $\left(\Delta_{2,3}^{2}+\Delta_{1,3}^{2}-\Delta_{1,2}^{2}\right)=0$ and so we get,

$$
b_{3}=\frac{-\left(x_{1}^{\prime \prime 2}+x_{2}^{\prime \prime 2}\right)}{2\left(\Delta_{1,3} x_{1}^{\prime \prime}+\Delta_{2,3} x_{2}^{\prime \prime}\right)} \text {. }
$$

Also, $b_{3}$ can be written in the form,

$$
b_{3}=\frac{-\left[g(N, N)+x_{3}^{\prime 2}\right]}{2\left[g(T, N) x_{3}^{\prime \prime}-g(N, N) x_{3}^{\prime}\right]} \text {. }
$$

In equation (7), when the parameterization is pseudo-arc so $g(N, N)=1, g(T, N)=0$ and we get,

$$
b_{3}=\frac{-\left(1+x_{3}^{\prime \prime 2}\right)}{2 x_{3}^{\prime}}, x_{3}^{\prime} \neq 0 \text {. }
$$

Where $\Delta_{2,3}=\left(x_{2}^{\prime} x_{3}^{\prime \prime}-x_{3}^{\prime} x_{2}^{\prime \prime}\right), \Delta_{1,3}=\left(x_{1}^{\prime} x_{3}^{\prime \prime}-x_{3}^{\prime} x_{1}^{\prime \prime}\right)$, and $\Delta_{1,2}=\left(x_{1}^{\prime} x_{2}^{\prime \prime}-x_{2}^{\prime} x_{1}^{\prime \prime}\right)$. Then we get,

$$
B(s)=\left(\frac{-1}{\Delta_{1,2}}\left(\Delta_{2,3} b_{3}+x_{2}^{\prime \prime}\right), \frac{1}{\Delta_{1,2}}\left(\Delta_{1,3} b_{3}+x_{1}^{\prime \prime}\right), \frac{-\left(1+x_{3}^{\prime \prime 2}\right)}{2 x_{3}^{\prime}}\right) \text {. }
$$

Where $\Delta_{1,2} \neq 0, x_{3}^{\prime} \neq 0$ with curvature $k=1$ and torsion $\tau=-\left\langle N^{\prime}, B\right\rangle=\frac{1}{2} g\left(\alpha^{\prime \prime \prime}, \alpha^{\prime \prime \prime}\right)$.

Example 3.1. Let $\alpha(s)=\frac{1}{r^{2}}(\cosh (r s), r s, \sinh (r s))$ if we calculate $1^{\text {st }}$ and $2^{\text {nd }}$ order derivatives (with respect to s) of $\alpha(s)$ and so $T(s)=\frac{1}{r}(\sinh (r s), 1, \cosh (r s))$. Since $\langle T, T\rangle=0$ so $\alpha(s)$ is a null curve and $N(s)=T^{\prime}(s)=(\cosh (r s), 0, \sinh (r s)) \operatorname{so}\langle N, N\rangle=1$, since $B(s)$ is unique light like vector such that $g(T, B)=1$ and it is orthogonal to $T$, by substituting in the equation (9). We get $B(s)=\frac{r}{2}(\sinh (r s)$, $1, \cosh (r s))$ and so $\langle B, B\rangle=0, N^{\prime}=r(\sinh (r s), 0, \cosh (r s))$. The pseudo torsion is $\tau=-\left\langle N^{\prime}, B\right\rangle=\frac{-r^{2}}{2}$ where $N$ is space like vector. Then $\alpha(s)$ is a null curve with curvature $k=1$ and the Frenet equations of $\alpha(s)$ are given by

$$
\left(\begin{array}{l}
T^{\prime} \\
N^{\prime} \\
B^{\prime}
\end{array}\right)=\left(\begin{array}{lll}
0 & k & 0 \\
\tau & 0 & k \\
0 & \tau & 0
\end{array}\right)\left(\begin{array}{l}
T \\
N \\
B
\end{array}\right)=\left(\begin{array}{ccc}
0 & 1 & 0 \\
\frac{-r^{2}}{2} & 0 & 1 \\
0 & \frac{-r^{2}}{2} & 0
\end{array}\right)\left(\begin{array}{c}
\frac{1}{r}(\sinh (r s), 1, \cosh (r s)) \\
(\cosh (r s), 0, \sinh (r s)) \\
\frac{r}{2}(\sinh (r s),-1, \cosh (r s))
\end{array}\right) .
$$

Corollary 3.1 Let $\xi(s)$ be a null curve in $\mathrm{E}_{1}^{3}$ with non-zero curvature and pseudo torsion $\tau$, then the bi-normal vector of $\xi(s)$ can be calculate by the form,

$$
B(s)=\left(\frac{1}{k}\right) N^{\prime}(s)-\left(\frac{\tau}{k}\right) T(s)=\left(\frac{1}{k}\right) \xi^{\prime \prime \prime}(s)-\left(\frac{\tau}{k}\right) \xi^{\prime}(s) .
$$

Such that $\tau=-g\left(N^{\prime}, B\right)$ or $\tau=\frac{1}{2} g\left(\xi^{\prime \prime \prime}, \xi^{\prime \prime \prime}\right)$.

Theorem 3.2. Let $\xi(s)$ be a null curve in $\mathrm{E}_{1}^{3}$ with non-zero curvature and pseudo torsion $\tau(s)$. Then $\xi(s)$ satisfies a vector differential fourth order as follow,

$$
\frac{d 4 \xi}{d s^{4}}-2 \tau\left(\frac{d 2 \xi}{d s^{2}}\right)-\tau^{\prime} \frac{d \xi}{d s}=0
$$


Proof. Since $\xi(s)$ be a null curve in $\mathrm{E}_{1}^{3}$ from the Frenet equation $(*)$. We get,

$$
T^{\prime}(s)=k N(s), N^{\prime}(s)=\tau T(s)+k B(s) \text { and } B^{\prime}(s)=\tau N(s),
$$

with $k=1$ and so we have,

$$
\begin{gathered}
T^{\prime \prime}(s)=N^{\prime}(s)=\tau T+B(s) \text { and } T^{\prime \prime \prime}(s)=\tau^{\prime} T+\tau T^{\prime}+B^{\prime}(s) . \text { Then, } \\
T^{\prime \prime \prime}(s)=\tau^{\prime} T+2 \tau T^{\prime} \text { and so } T^{\prime \prime \prime}(s)-2 \tau T^{\prime}-\tau^{\prime} T=0 \text { denoting } T=\frac{d \xi}{d s}, \\
\frac{d 4 \xi}{d s^{4}}-2 \tau\left(\frac{d 2 \xi}{d s^{2}}\right)-\tau^{\prime} \frac{d \xi}{d s}=0 .
\end{gathered}
$$

\section{Folding of Null Curves}

Theorem 4.1. Let $\xi(s)$ be a null curve in $\mathrm{E}_{1}^{3}$ with non-zero curvature and $\Psi(s)=f(\xi(s))$ be a topological folding of $\xi(s)$ for all $s$ where $s \in$ Domain $(\Psi(s))=I \subset \operatorname{Domain} \xi(s)$ defined by frame vectors. Then $\Psi(s)=f(\xi(s))$ is a null curve and the Frenet apparatus of the folded curve $\Psi(s)$ can be formed by the Frenet apparatus of $\xi(s)$.

Proof. Let $\xi=\xi(s)$ be a null curve in $E_{1}^{3}$ with non-zero curvature and $\Psi(s)=f(\xi(s)), s \in I \subset$ domain $\xi(s)$ is a topological folding of $\xi(s)$ with curvatures $k_{f}$ and $\tau_{f}$ and so,

$\Psi(s)=f(\xi(s)), \Psi^{\prime}(s)=f^{\prime}(\xi) \xi^{\prime}(s)=f^{\prime}(\xi) T(s)$. And we get, $\left\langle\Psi^{\prime}, \Psi^{\prime}\right\rangle=\left\langle f^{\prime} \xi^{\prime}(s), f^{\prime} \xi^{\prime}(s)\right\rangle=f^{\prime 2}\langle T(s), T(s)\rangle=0$. Since $\xi(s)$ is a null curve with $\langle T(s), T(s)\rangle=0, f^{\prime 2}>0$ for all $s$. Then $\Psi(s)$ is a null curve with curvatures $k_{f}=k=1$ and $T_{f}=f^{\prime}(s) T(s)$ where,

$\Psi^{\prime \prime \prime}(s)=T^{3} f^{\prime \prime \prime}(\xi)+3 T N f^{\prime \prime}(\xi)+f^{\prime}(\xi) \xi^{\prime \prime \prime}(s)$.

By substituting the value of $\xi^{\prime \prime \prime}(s)$ from the Frenet apparatus of the curve $\xi(\mathrm{s})$ in corollary 3.1. Then,

$T_{f}=T(s) f^{\prime}(\xi)$,

$N_{f}=\Psi^{\prime \prime}(s)=N(s) f^{\prime}(\xi)+T^{2}(s) f^{\prime \prime}(\xi)$,

$$
B_{f}=\Psi^{\prime \prime \prime}(s)=f^{\prime}(\xi) B(s)+T^{3} f^{\prime \prime \prime}(\xi)+3 T N f^{\prime \prime}(\xi), \tau_{f}=\tau=0,
$$

$B_{f}=\Psi^{\prime \prime \prime}-\tau_{f} \Psi^{\prime}=\left(\tau-\tau_{f}\right) f^{\prime}(\xi) T+f^{\prime}(\xi) B+T^{3} f^{\prime \prime \prime}(\xi)+3 T N f^{\prime \prime}(\xi)$, for all $\tau \neq 0$ and $\tau_{f} \neq 0$.

Corollary 4.1. Let $\xi(s)$ be a null curve in $E_{1}^{3}$ and $\Psi(s)=f(\xi(s))$ be a topological folding of $\xi(s)$. Then the limit of folding's of $\xi(s)$ is a null point.

Proof. Let $\Psi(s)=f(\xi(s))$ be a topological folding of the null curve $\xi(s)$ in $E_{1}^{3}$ so $\Psi(s)$ be null curve and we have,

$$
\begin{gathered}
\Psi_{1}(s): f(\xi(s)) \rightarrow f(\xi(s)), \Psi_{2}(s): \Psi_{1}(f(\xi(s))) \rightarrow \Psi_{1}(f(\xi(s))), \\
\Psi_{3}(s): \Psi_{2}\left(\Psi_{1}(f(\xi(s)))\right) \rightarrow \Psi_{2}\left(\Psi_{1}(f(\xi(s)))\right), \ldots, \\
\left.\left.\Psi_{n}: \Psi_{(n-1)}\left(\Psi_{(n-2)}\left(\ldots \Psi_{1} f(\xi)\right)\right)\right) \ldots\right) \rightarrow \Psi_{(n-1)}\left(\Psi_{(n-2)}\left(\ldots \Psi_{1}(f(\xi))\right) \ldots\right)
\end{gathered}
$$

Then $\lim _{n \rightarrow \infty} \Psi_{n}=p=(0,0,0)$, which is a null point.

Definition 4.1. Let $\left.\xi(s)=\left\{x_{1}(s), x_{2}(s), x_{3}(s)\right)\right\}$ be a null curve in $E_{1}^{3}$. Then $\Psi(\mathrm{s})$ be an isometric folding defined as follows,

$\left.\Psi(\mathrm{s}): \xi(\mathrm{s})=\left\{x_{1}(s), x_{2}(s), x_{3}(s)\right)\right\} \rightarrow \xi_{f}=\left\{\left(\frac{\left|x_{1}(s)\right|}{m}, \frac{\left|x_{2}(s)\right|}{m}, \frac{\left|x_{3}(s)\right|}{m}\right)\right\}$ for all $s,|m|>1, m \neq 0$.

Theorem 4.2. Let $\xi(s)=\left(x_{1}(s), x_{2}(s), x_{3}(s)\right)$ be a null curve in $E_{1}^{3}$ and $\Psi(\xi)=\left(\frac{\left|x_{1}(s)\right|}{m}, \frac{\left|x_{2}(s)\right|}{m}, \frac{\left|x_{3}(s)\right|}{m}\right)$ for all $s$ be an isometric folding of $\xi(s),|m|>1$. Then the folding $\Psi(s)$ be a null curve and, $\left(\begin{array}{l}T_{f} \\ N_{f} \\ B_{f}\end{array}\right)=$ $\left(\begin{array}{ccc}\frac{\delta}{m} & 0 & 0 \\ 0 & \frac{\delta}{m} & 0 \\ \delta m & 0 & 0\end{array}\right)\left(\begin{array}{l}T \\ N \\ B\end{array}\right), \delta=1$ if $x_{i}(s)>0$ and $\delta=-1$ if $x_{i}(s)<0, i \in\{1,2,3\}$ 
Proof. Let $\Psi(\xi): \xi(s)=\left(x_{1}(s), x_{2}(s), x_{3}(s)\right) \rightarrow\left(\frac{\left|x_{1}(s)\right|}{m}, \frac{\left|x_{2}(s)\right|}{m}, \frac{\left|x_{3}(s)\right|}{m}\right),|m|>1$, be an isometric folding of the null curve $\xi(s)=\left(x_{1}(s), x_{2}(s), x_{3}(s)\right) \quad$ in $\quad E_{1}^{3}$. If $\quad x_{i}(s)>0, \quad i \in \quad\{1,2,3\}$, then $\Psi^{\prime}=\frac{d \Psi}{d s}=\frac{1}{m}\left(x_{1}^{\prime}(s), x_{2}^{\prime}(s), x_{3}^{\prime}(s)\right)$, since $\xi(s)$ be a null curve where $\langle T(s), T(s)\rangle=0$ and $\left\langle T^{\prime}(s), T^{\prime}(s)\right\rangle=0$, for the folded curve $\xi_{f}(s)=\left(\frac{x_{1}(s)}{m}, \frac{x_{2}(s)}{m}, \frac{x_{3}(s)}{m}\right)$ since $\left\langle T_{f}(s), T_{f}(s)\right\rangle=\frac{1}{m^{2}}\langle T(s), T(s)\rangle=0 \quad$ and $\left\langle T_{f}{ }^{\prime}(s), T_{f}{ }^{\prime}(s)\right\rangle=\frac{1}{m^{2}}\left\langle T^{\prime}(s), T^{\prime}(s)\right\rangle=0$, then the folded curve $\xi_{f}(s)$ is a null curve. Since $B(s)$ is unique light like vector, also $g(T, B)=-1$ and $B$ is orthogonal to $N$. Then,

$T_{f}(\mathrm{~s})=\Psi^{\prime}(s)=\frac{1}{m} T(s), N_{f}(\mathrm{~s})=T_{f}^{\prime}=\frac{1}{m} T^{\prime}(s)=\frac{1}{m} N(s)$ and from theorem(1), we get,

$B_{f}(\mathrm{~s})=m B(s)$.

If $x_{i}(s)<0, \quad i \in\{1,2,3\}$ and $\xi_{f}(s)=\left(\frac{-x_{1}(s)}{m}, \frac{-x_{2}(s)}{m}, \frac{-x_{3}(s)}{m}\right)$, so $T_{f}(\mathrm{~s})=\frac{-1}{m} T(s), \quad N_{f}(\mathrm{~s})=\frac{-1}{m} N(s)$ and $B_{f}(\mathrm{~s})=-m B(s)$. Then the Frenet apparatus of the folding $\Psi(\xi)$ can be formed by the Frenet apparatus of $\xi(s)$.

Now we introduce a type of folding which make the null curves to be space like curves and time like curves and the converse as follows,

\section{Conditional Fractal Folding of Null Curves}

Definition 5.1 Let $\xi(s)$ be any curve in $\mathrm{E}_{1}^{n}$ the map which is defined as $\xi_{f}:\left(x_{1}(s), x_{2}(s), \ldots, x_{i}(s), \ldots, x_{n}\right) \rightarrow$ $\left(x_{1}(s), x_{2}(s), \ldots, \varepsilon x_{i}(s), \ldots, x_{n}(s)\right)$ for $\varepsilon \leq 1, \varepsilon \neq 0$ is called conditional fractal folding of the coordinates $x_{i}, \varepsilon$ depends on the type of the curve $\xi_{f}$ (space like, time like and null curve) (M. EL-Ghoul \& A. M. Soliman. 2002).

Theorem 5.1. Let $\xi(\mathrm{s})$ be a null curve in $E_{1}^{3}$. Under the conditional fractal folding $\Psi(\mathrm{s}): \xi(\mathrm{s})=$ $\left.\left\{x_{1}(s), x_{2}(s), x_{3}(s)\right)\right\} \rightarrow \xi_{f}=\left(x_{1}(s), x_{2}(s), \varepsilon x_{3}(s)\right), \varepsilon \neq 0$ for all $s$, then $\xi_{f}$ is space like curve if $|\varepsilon|<1, \xi_{f}$ is null curve if $\varepsilon= \pm 1$ and $\xi_{f}$ is time like curve if $|\varepsilon|>1$.

Proof. Let $\left.\xi(\mathrm{s})=\left\{x_{1}(s), x_{2}(s), x_{3}(s)\right)\right\}$ be a null curve in $E_{1}^{3},\langle T, T\rangle=0$, so $x_{1}{ }^{2}+x_{2}{ }^{2}=x_{3}{ }^{2}$ and $\Psi(\mathrm{s})$ be conditional folding defined as $\Psi(\mathrm{s}): \xi(\mathrm{s}) \rightarrow \xi_{f}$, if $\xi_{f}=\left(x_{1}(s),\left(x_{2}(s), \varepsilon x_{3}\right), \varepsilon \neq 0\right.$, so $\left\langle T_{f}, T_{f}\right\rangle=x_{1}{ }^{2}+$ $x_{2}{ }^{2}-\varepsilon^{2} x_{3}{ }^{2}$ and then let $g(s)=\left\langle T_{f}, T_{f}\right\rangle$, then we have $g^{\prime}(s)=2\left\langle T_{f}, T_{f}^{\prime}\right\rangle=2\left\langle T_{f}, k_{f} N_{f}\right\rangle=0$ where $k_{f} \neq 0$ is constant, so $g^{\prime}(s)=0$ and $g(s)=c_{1}, c_{1}$ is constant.

If $c_{1}>0,\left\langle T_{f}, T_{f}\right\rangle>0$ and $x_{1}{ }^{2}+x_{2}{ }^{2}-\varepsilon^{2} x_{3}{ }^{2}>0$ so $x_{3}{ }^{2}\left(1-\varepsilon^{2}\right)>0, \varepsilon^{2}<1$, then $\xi_{f}$ is space- like if $|\varepsilon|<1$.

If $c_{1}<0$ we have $\left\langle T_{f}, T_{f}\right\rangle<0$ and $\varepsilon^{2}>1$, then $\xi_{f}$ is time like curve if $|\varepsilon|>1$.

If $c_{1}=0,\left\langle T_{f}, T_{f}\right\rangle=0$ and so $\varepsilon^{2}=1$, then $\xi_{f}$ is null curve if $\varepsilon= \pm 1$.

Corollary 5.1. Let $\xi(\mathrm{s})$ be a null curve in $E_{1}^{3}$. Under the conditional fractal folding which is defined as, $\left.\Psi(\mathrm{s}): \xi(\mathrm{s})=\left\{x_{1}(s), x_{2}(s), x_{3}(s)\right)\right\} \rightarrow \xi_{f}=\left(x_{1}(s), x_{2}(s), \varepsilon x_{3}(s)\right)$ for all $\varepsilon \leq 1, \varepsilon \neq 0$.

The Frenet equations of the folded curve $\xi_{f}$ is depends on $\varepsilon$.

Corollary 5.2. Let $\xi(\mathrm{s})$ be a null curve in $E_{1}^{3}$ and $\Psi(\mathrm{t})$ be conditional fractal folding defined as $\Psi(\mathrm{s})$ : $\left.\xi(\mathrm{s})=\left\{x_{1}(s), x_{2}(s), x_{3}(s)\right)\right\} \rightarrow \xi_{f}$ and $\xi_{f}=\left(\varepsilon x_{1}(s), \varepsilon x_{2}(\mathrm{~s}), x_{3}(s)\right), \varepsilon \neq 0, \varepsilon \leq 1$ for all $s$. Then

$\xi_{f}$ is space like curve if $|\varepsilon|>1, \xi_{f}$ is time like curve if $|\varepsilon|<1$. and $\xi_{f}$ is null curve if $\varepsilon= \pm 1$.

Corollary 5.3. Let $\left.\xi(\mathrm{s})=\left\{x_{1}(s), x_{2}(s), x_{3}(s)\right)\right\}$ be any curve in $E_{1}^{3}$ under the conditional fractal folding $\Psi(\mathrm{s}): \xi(\mathrm{s}) \rightarrow \xi_{f}, \xi_{f}=\left(\varepsilon x_{1}(s), x_{2}(\mathrm{~s}), x_{3}(s)\right)$ or $\xi_{f}=\left(x_{1}(s), \varepsilon x_{2}(\mathrm{~s}), x_{3}(s)\right), \quad \varepsilon \neq 0,|\varepsilon|<1$, for all $s$. Then the limit of a sequence of foldings of $\xi(\mathrm{s})$ is never being null curve.

Proof. Let the limit of a sequence of foldings of any curve $\xi(\mathrm{s})$ in $E_{1}^{3}$ be a null curve with $\xi_{f}=\left(0, x_{2}(s), x_{3}(s)\right)$, or $\xi_{f}=\left(x_{1}(s), 0, x_{3}(s)\right)$ and $\xi_{f}=\left(x_{1}(s), x_{2}(s), 0\right)$, then from theorem 3.1, the bi-normal vector of the folded curve $B_{f}$ undefined, also $N_{f}^{\prime}=\tau T_{f}-k B_{f}$ undefined. The Frenet equations of $\xi_{f}$ cannot appoints and so this contradict with $\xi_{f}$ be null curve. Then $\xi_{f}$ never being null curve.

Theorem 5.2. Let $\left.\xi(\mathrm{s})=\left\{x_{1}(s), x_{2}(s), x_{3}(s)\right)\right\}$ be a null curve in $E_{1}^{3}$. Then the conditional folding $\xi_{f}=$ $\left(\varepsilon x_{1}(s), \varepsilon x_{2}(s), \varepsilon x_{3}(s)\right),|\varepsilon|<1$, of $\xi(\mathrm{s})$ be null curve. And the Frenet equations of the folded curve $\xi_{f}$ can be formed by the Frenet equations of $\xi(\mathrm{s})$. 
Proof. Let $\left.\xi(s)=\left\{x_{1}(s), x_{2}(s), x_{3}(s)\right)\right\}$, be null curve in $E_{1}^{3}$ and $\xi_{f}=\left(\varepsilon x_{1}(s), \varepsilon x_{2}(s), \varepsilon x_{3}(s)\right),|\varepsilon|<1$ be a conditional fractal folding of $\xi(s)$ and so $\left\langle T_{f}, T_{f}\right\rangle=\varepsilon^{2}\langle T(s), T(s)\rangle=0$. Then the folded curve $\xi_{f}$ is null curve, with curvature $k_{f}=k=1$ and torsion $\tau_{f}=\tau$, by using the form of Frenet equations in theorem 1 . Then we have,

$$
\left.\begin{array}{rl}
T_{f}(\mathrm{~s}) & =\varepsilon T(s) \\
N_{f}(\mathrm{~s}) & =\varepsilon N(s) \\
B_{f}(\mathrm{~s}) & =\left(\frac{1}{\varepsilon}\right) B(s)
\end{array}\right\} .
$$

Corollary 5.4. Let $\xi(\mathrm{s})=\left\{\left(x_{1}(s), x_{2}(s), x_{3}(s)\right)\right\}$ be a null curve in $E_{1}^{3}$. Then the conditional fractal folding $\xi_{f}=\left(\varepsilon_{i} x_{1}(s), \varepsilon_{i} x_{2}(s), \varepsilon_{i} x_{3}(s)\right), i \in \mathbb{N},\left|\varepsilon_{i}\right|<1, \varepsilon_{i} \neq 0$ be a null curve and the limit of a sequence of foldings of a null curve $\xi(\mathrm{s})$ be a null point.

Proof. Let $\left.\xi(s)=\left\{x_{1}(s), x_{2}(s), x_{3}(s)\right)\right\}$ be a null curve in $E_{1}^{3}$. So $\langle T(s), T(s)\rangle$, since $\left\langle T_{f}(s), T_{f}(s)\right\rangle=$ $\varepsilon_{i}^{2}\langle T(s), T(s)\rangle=0, \varepsilon_{i} \neq 0$, then $\xi_{f}$ is a null curve.

Let $f: \xi \rightarrow \varepsilon_{i} \xi$ be a conditional fractal folding of the null curve $\xi$ such that $\forall x, y \in \xi, d(x, y)$ $\geq d(f(x), f(y))$ where $\xi(\mathrm{s})$ be a null curve. By successive steps of conditional fractal folding's we get,

$$
\begin{gathered}
f_{1}: \xi \rightarrow \varepsilon_{1} \xi,\left|\varepsilon_{1}\right|<1, \\
f_{2}: \varepsilon_{1} \xi \rightarrow \varepsilon_{2}(\xi), \varepsilon_{2}<\varepsilon_{1}, \\
f_{3}: \varepsilon_{2} \xi \rightarrow \varepsilon_{3}(\xi), \varepsilon_{3}<\varepsilon_{2} \cdots, \\
f_{n}: \varepsilon_{(n-1)} \xi \rightarrow \varepsilon_{n}(\xi), \varepsilon_{n}<\varepsilon_{(n-1)} \ll 1,
\end{gathered}
$$

$\lim _{n \rightarrow \infty} f_{n}(\xi)=p$ where $p=(0,0,0)$ is a null point.

Theorem 5.5. If $\xi(s)$ and $\bar{\xi}(s)$ are null curves with non-zero curvature in $\mathrm{E}_{1}^{3}$ and $F_{\tau}: \xi \rightarrow \bar{\xi}$ is an isotorsion folding, then the torsion of $\bar{\xi}$ identically zero if and only if $\xi$ is a part of the null cubic.

Proof. Let $\bar{\xi}$ be a null curve in $E_{1}^{3}$ has torsion identically zero. Since $F_{\tau}$ is an isotorsion folding from $\xi$ into $\bar{\xi}$. Then the torsion of $\xi$ is zero and the Maclaurin series can be written as,

$\xi(s)=\xi(0)+\xi^{\prime}(0) s+\xi^{\prime \prime}(0) \frac{s^{2}}{2}+\xi^{\prime \prime \prime}(0) \frac{s^{3}}{6}$.

Since $B(s)=-\xi^{\prime \prime \prime}(s)$ when $\tau=0$. So we get,

$\xi(s)=\xi(0)+T(0) s+N(0) \frac{s^{2}}{2}-B(0) \frac{s^{3}}{6}$. With Frenet frame $\{T, N, B\}$ of $\xi(s)$ in this case $g(T, T)=$ $g(B, B)=0, g(T, B)=g(N, N)=1$. Without loss of generality,

assume that $T(0)=\frac{1}{\sqrt{2}}(1,0,1), N(0)=(0,1,0)$ and $B(0)=\frac{1}{\sqrt{2}}(1,0,-1)$ so we get,

$\xi(s)=\frac{1}{6 \sqrt{2}}\left(6 s-s^{3}, 3 \sqrt{2} s^{2}, 6 s+s^{3}\right)$. Then $\xi(s)$ is a part of null cubic. Conversely let the curve $\xi(s)$ be a part of the null cubic, then the torsion of $\xi(s)$ identically zero. Since $\mathrm{F}_{\tau}$ is an isotorsion folding and $\bar{\xi}$ has torsion identically zero.

\section{Conditional Deformations of Null Curves in $E_{1}^{3}$}

Theorem 6.1. Let $\xi(s)$ be a null curve in $E_{1}^{3}$ and $F(x)=M x+c, c \in \mathbb{R}, M \neq 0$ be a conditional deformation of $\xi(s)$ defined as $\mathrm{F}(s)=\left(M x_{1}(\mathrm{~s})+\mathrm{c}, \mathrm{M} x_{2}(s)+c, \mathrm{M} x_{3}(s)+c\right)$.Then the deformation $\mathrm{F}(s)$ be a null curve and,

$$
\left(\begin{array}{l}
T_{F} \\
N_{F} \\
B_{F}
\end{array}\right)=\left(\begin{array}{ccc}
M & 0 & 0 \\
0 & M & 0 \\
0 & 0 & \frac{1}{M}
\end{array}\right)\left(\begin{array}{l}
T \\
N \\
B
\end{array}\right) .
$$

Proof. $\operatorname{Let} \xi(s)$ be a null curve in $E_{1}^{3}$ and $F(s)$ be a conditional deformation of $\xi(s)$ defined as $F(s)=$ $M \xi(s)+c$, since $\xi(s)$ is a null curve so $\left\langle\xi^{\prime}, \xi^{\prime}\right\rangle=0, M \neq 0$ we get,

$F^{\prime}(\mathrm{s})=\mathrm{M} \xi^{\prime}(s),\left\langle F^{\prime}, F^{\prime}\right\rangle=M^{2}\left\langle\xi^{\prime}, \xi^{\prime}\right\rangle=0$. Then $F(s)$ is a null curve with $k=k_{d}=1$ and we get,

$T_{F}(s)=F^{\prime}(s)=M \xi^{\prime}(s)=M T(s)$,

$N_{F}(s)=T_{F}{ }^{\prime}(s)=M T^{\prime}(s)=M N(s)$, 
$B_{F}(s)=\frac{1}{M} B$, where the torsion be $\tau_{F}=-\left\langle N_{F}{ }^{\prime}, B_{F}\right\rangle=\tau$. Then the Frenet apparatus of $F(s)$ can be formed by the Frenet apparatus of $\xi(s)$.

Corollary 6.1. A null curve $\xi(s)$ in $E_{1}^{3}$ under the conditional deformation $F(s)=M \xi(s)+c$ of $\xi(s), M \neq 0$ has the first curvature identically zero if and only if $F(\xi)$ be a part of a straight line.

Proof. Assume that the conditional deformation $F(\xi)$ of the null curve $\xi(s)$ be $\mathrm{F}(s)=\left(M x_{1}(\mathrm{~s})+\right.$ $\left.\mathrm{c}, \mathrm{M} x_{2}(s)+c, \mathrm{M} x_{3}(s)+c\right)$ where $M \neq 0$ such that $\operatorname{dim} F(\xi)=\operatorname{dim} \xi(s)$ and from the Frenet equations with first curvature $\mathrm{k}=0$, then $F^{\prime \prime}(\xi)=M N(s)=0$ and this implies that $F(\xi)$ is a straight line, where $k_{F}=\left\|T_{F}^{\prime}(s)\right\|, N_{F}(s)=T_{F}^{\prime}(s)$. Conversely, let $F(\xi)$ be a straight line then $F^{\prime \prime}(\xi)=M N(s)=0$ and $F(\xi)$ has the curvature $k_{F}$ which is identically zero.

Remark 6.1. If $\alpha(\mathrm{s})$ be a light-like curve in $\mathrm{E}_{1}^{3}$ with standard flat metric $g=-d \bar{x}^{2}+d \bar{y}^{2}+d \bar{z}^{2}, g(N, N)>$ 0 , when the parameterization is pseudo arc so $g(N, N)=1$ with $g(T, T)=0, g(B, B)=0$ and $g(T, N)=$ 0 , and $B(s)$ is unique light like vector such that $g(T, B)=1$ and it is orthogonal to $N$ the pseudo torsion of $\alpha(\mathrm{s})$ be $\tau=-\left\langle N^{\prime}, B\right\rangle$, then the Frenet equations of $\alpha(\mathrm{s})$ are

$$
\left(\begin{array}{l}
T^{\prime} \\
N^{\prime} \\
B^{\prime}
\end{array}\right)=\left(\begin{array}{ccc}
0 & k & 0 \\
\tau & 0 & -k \\
0 & -\tau & 0
\end{array}\right)\left(\begin{array}{l}
T \\
N \\
B
\end{array}\right) .10 .
$$

Where the curvature $k$ can take only two values 0 when $\alpha$ is a straight null line or 1 in all other cases.

Theorem 6.2. Let $\xi(s)$ be a null curve in $\mathrm{E}_{1}^{3}$ with standard flat metric $g=d x^{2}+d y^{2}-d z^{2}$. Under the conditional deformation,

$D: \quad \xi(s)=(x(s), y(s), z(s)) \rightarrow \quad D(\xi)=(\bar{x}(s), \bar{y}(s), \bar{z}(s)) \quad=(z(s), y(s),-x(s))$ which rotation the coordinates $x$ and $z$ in x-z plane with rotation angle $\theta=\frac{n \pi}{2}, n \in \mathbb{R}, n$ is odd integer. Then $D(\xi)$ be a null curve with standard flat metric $g=-d \bar{x}^{2}+d \bar{y}^{2}+d \bar{z}^{2}$.

Proof. Let $\xi(s)$ be a null curve in $\mathrm{E}_{1}^{3}$ with standard flat metric $g=d x^{2}+d y^{2}-d z^{2}$ since the equation of which rotation coordinates $x$ and $z$ in x-z plane can be written as,

$\left(\begin{array}{l}\bar{x} \\ \bar{z}\end{array}\right)=\left(\begin{array}{cc}\cos \theta & \sin \theta \\ -\sin \theta & \cos \theta\end{array}\right)\left(\begin{array}{l}x \\ z\end{array}\right), \bar{y}=y$. Under the conditional deformation $D(\xi)$ which rotation coordinates $x$ and $z$ with rotation angle $\theta=\frac{n \pi}{2}$, and $n \in \mathbb{R}, n$ is odd integer then $\left(\begin{array}{l}\bar{x} \\ \bar{z}\end{array}\right)=\left(\begin{array}{cc}0 & 1 \\ -1 & 0\end{array}\right)\left(\begin{array}{l}x \\ z\end{array}\right), \bar{y}=y$, or $\left(\begin{array}{l}\bar{x} \\ \bar{z}\end{array}\right)=$ $\left(\begin{array}{cc}0 & -1 \\ 1 & 0\end{array}\right)\left(\begin{array}{l}x \\ z\end{array}\right), \bar{y}=y$

$D(\xi)=(\bar{x}(s), \bar{y}(s), \bar{z}(s))=(z(s), y(s),-x(s))$ also,

$g\left(D^{\prime}, D^{\prime}\right)=-d \bar{x}^{2}+d \bar{y}^{2}+d \bar{z}^{2}$,

$\left\langle D^{\prime}, D^{\prime}\right\rangle=-d \bar{x}^{2}+d \bar{y}^{2}+d \bar{z}^{2}=d x^{2}+d y^{2}-d z^{2}=\left\langle\xi^{\prime}, \xi^{\prime}\right\rangle=0$. Then the conditional deformation $D(\xi)$ be a null curve with standard flat metric $g=-d \bar{x}^{2}+d \bar{y}^{2}+d \bar{z}^{2}$.

Theorem 6.3. Let $\xi(s)$ be a null curve in $\mathrm{E}_{1}^{3}$ with the standard flat metric given by $g=-d x_{1}^{2}+d x_{2}^{2}+d x_{3}^{2}$. Then the bi-normal vector of can be calculated by,

$B(s)=\left(\frac{1}{\Delta_{1,2}}\left(x_{2}^{\prime \prime}-\Delta_{2,3} b_{3}\right), \frac{1}{\Delta_{1,2}}\left(\Delta_{1,3} b_{3}-x_{1}^{\prime \prime}\right), \frac{-\left(1+x_{3}^{\prime \prime 2}\right)}{2 x_{3}^{\prime}}\right), \Delta_{1,2} \neq 0, x_{3}^{\prime} \neq 0$.

Where $\Delta_{2,3}=\left(x_{2}^{\prime} x_{3}^{\prime \prime}-x_{3}^{\prime} x_{2}^{\prime \prime}\right), \Delta_{1,3}=\left(x_{1}^{\prime} x_{3}^{\prime \prime}-x_{3}^{\prime} x_{1}^{\prime \prime}\right)$ and $\Delta_{1,2}=\left(x_{1}^{\prime} x_{2}^{\prime \prime}-x_{2}^{\prime} x_{1}^{\prime \prime}\right)$.

Proof. Let $\xi(s)=\left(x_{1}(s), x_{2}(s), x_{3}(s)\right)$ be a null curve in $\mathrm{E}_{1}^{3}$ with tangent vector $T(s)=\left(x_{1}^{\prime}(s), x_{2}^{\prime}(s)\right.$, $\left.x_{3}^{\prime}(s)\right)$ and the normal vector $N(s)=T^{\prime}(s)=\left(x_{1}^{\prime \prime}(s), x_{2}^{\prime \prime}(s), x_{3}^{\prime \prime}(s)\right)$, to calculate the bi-normal vector of the curve $\xi(s)$, let $B(s)=\left(b_{1}, b_{2}, b_{3}\right)$, since $B(s)$ is unique light-like vector. Then,

$\langle B, B\rangle=0$ and we get,

$$
-b_{1}{ }^{2}+{b_{2}}^{2}+{b_{3}}^{2}=0 \text {. }
$$

Also since $g(T, B)=1$ we get,

$$
-x_{1}^{\prime} b_{1}+x_{2}^{\prime} b_{2}+x_{3}^{\prime} b_{3}=1 \text {. }
$$

Since $B$ be orthogonal to $N$, then $\langle N, B\rangle=0$ and we get,

$$
-x_{1}^{\prime \prime} b_{1}+x_{2}^{\prime \prime} b_{2}+x_{3}^{\prime \prime} b_{3}=0 \text {. }
$$

By solving these equations as theorem1, we get the bi-normal vector be 


$$
B(s)=\left(\frac{1}{\Delta_{1,2}}\left(\Delta_{2,3} b_{3}+x_{2}^{\prime \prime}\right), \frac{1}{\Delta_{1,2}}\left(\Delta_{1,3} b_{3}+x_{1}^{\prime \prime}\right), \frac{\left(1-x_{3}^{\prime \prime 2}\right)}{2 x_{3}^{\prime}}\right), \Delta_{1,2} \neq 0, x_{3}^{\prime} \neq 0 .
$$

Where $\Delta_{2,3}=\left(x_{2}^{\prime} x_{3}^{\prime \prime}-x_{3}^{\prime} x_{2}^{\prime \prime}\right), \Delta_{1,3}=\left(x_{1}^{\prime} x_{3}^{\prime \prime}-x_{3}^{\prime} x_{1}^{\prime \prime}\right)$ and $\Delta_{1,2}=\left(x_{1}^{\prime} x_{2}^{\prime \prime}-x_{2}^{\prime} x_{1}^{\prime \prime}\right)$ and so,

$$
b_{1}=\frac{1}{\Delta_{1,2}}\left(\Delta_{2,3} b_{3}+x_{2}^{\prime \prime}\right), b_{2}=\frac{1}{\Delta_{1,2}}\left(\Delta_{1,3} b_{3}+x_{1}^{\prime \prime}\right) \text { and } b_{3}=\frac{\left(x_{2}^{\prime \prime 2}-x_{1}^{\prime \prime 2}\right)}{2\left(\Delta_{1,3} x_{1}^{\prime \prime}-\Delta_{2,3} x_{2}^{\prime \prime}\right)} \text {. }
$$

Also $b_{3}$ can be written in the form,

$$
b_{3}=\frac{\left[g(N, N)-x_{3}^{\prime \prime 2}\right]}{2\left[g(N, N) x_{3}^{\prime}-g(T, N) x_{3}^{\prime \prime}\right]} .
$$

In equation (15), when the parameterization is pseudo-arc so $g(N, N)=1, g(T, N)=0$. Then,

$$
b_{3}=\frac{\left(1-x_{3}^{\prime \prime 2}\right)}{2 x_{3}^{\prime}}, x_{3}^{\prime} \neq 0 .(16)
$$

Example 6.1. Let $\alpha(s)=\frac{1}{r^{2}}(\cosh (r s), r s, \sinh (r s))$ be a null curve in $\mathrm{E}_{1}^{3}$ with standard flat metric $g=d x^{2}+d y^{2}-d z^{2}$ and $\alpha_{D}(s)=\frac{1}{r^{2}}(\sinh (r s), r s, \cosh (r s))$ be deformation of the null curve $\alpha(s)$ by rotation coordinates $x$ and $z$ with rotation angle $\theta=\frac{n \pi}{2}, n$ is odd integer with standard flat metric $g=$ $-d \bar{x}^{2}+d \bar{y}^{2}+d \bar{z}^{2}$. If we calculate $1^{\text {st }}$ and $2^{\text {nd }}$ order derivatives (with respect to s) of $\alpha_{D}(s)$ and so $T(s)=$ $\frac{1}{r}(\cosh (r s), 1, \sinh (r s))$, since $\langle T, T\rangle=0$ so $\alpha(s)$ is null a curve and $N(s)=T^{\prime}(s)=$ $(\sinh (r s), 0, \cosh (r s))$, so $\langle N, N\rangle=1$, since $B(s)$ is unique light like vector such that $g(T, B)=1$ and it is orthogonal to $T$ by substituting in the equation (13). Then $B(s)=\frac{-r}{2}(\cosh (r s),-1, \sinh (r s))$, so $\langle B, B\rangle=0, N^{\prime}=r(\cosh (r s), 0, \sinh (r s))$, the pseudo torsion is $\tau=-\left\langle N^{\prime}, B\right\rangle=\frac{1}{2} g\left(\alpha^{\prime \prime \prime}, \alpha^{\prime \prime \prime}\right)=\frac{-r^{2}}{2}, N$ is space like vector. Then $\alpha(s)$ is a null curve with curvature $k=1$ and the Frenet equations of $\alpha(s)$ are given by

$$
\left(\begin{array}{l}
T^{\prime} \\
N^{\prime} \\
B^{\prime}
\end{array}\right)=\left(\begin{array}{ccc}
0 & k & 0 \\
\tau & 0 & -k \\
0 & -\tau & 0
\end{array}\right)\left(\begin{array}{l}
T \\
N \\
B
\end{array}\right)=\left(\begin{array}{ccc}
0 & 1 & 0 \\
\frac{-r^{2}}{2} & 0 & -1 \\
0 & \frac{r^{2}}{2} & 0
\end{array}\right)\left(\begin{array}{c}
\frac{1}{r}(\cosh (r s), 1, \sinh (r s)) \\
(\sinh (r s), 0, \cosh (r s)) \\
\frac{-r}{2}(\cosh (r s),-1, \sinh (r s))
\end{array}\right)
$$

Corollary 6.2. Under the conditional deformation which is defined by,

$D: \xi(s)=(x(s), y(s), z(s)) \rightarrow D(\xi)=(z(s), y(s), x(s))$, the Frenet equations of $D(\xi)$ are invariant.

Proof. The proof is clear from theorem 6.3, the Frenet equations of $D(\xi)$ calculates from equation (10).

\section{References}

A. E. El-Ahmady. (2007). The variation of the density on chaotic spheres in chaotic space-like Minkowski space time, Chaos. Solutions and Fractals, 31, 1272-1278. https://doi.org/10.1016/j.chaos.2005.10.112

A. E. El-Ahmady \& A. El-Araby. (2010). On fuzzy spheres in fuzzy Minkowski space. Nuovo Cimento, 125 B.

A. E. El-Ahmady \& A.T.M. Zidan. (2018). On hyperbola in Minkowski3-space and its deformations. International Journal of Applied Mathematics and Statistics, 57(5),115-127.

A. E. El-Ahmady \& A.T.M. Zidan. (2019). On the retraction of pseudo null space-like curves in Minkowski 3-space. JP Journal of Geometry and Topology, 23(2), 89-106. https://doi.org/10.17654/GT023020089

A. E. El-Ahmady \& E. Al-Hesiny. (2013) On the geometry of curves in Minkowski 3-space and its folding. Applied Mathematics, 4(5), 746-752. https://doi.org/10.4236/am.2013.45103

J. Walrave. (1995). Curves and surfaces in Minkowski space, Ph.D. Thesis, ProQuest LLC, AnnArbor, Mich, USA, Katholieke Universiteit, Leuven, Belgium.

M. EL-Ghoul \& A. M. Soliman. (2002). Retraction of Chaotic Manifolds and Fractal. LE MATEMATICHE, LVII Fasc(II), 229-240.

M. P. Docarmo. (1992). Riemannian geometry. Boston: Birkhauser. https://doi.org/10.1007/978-1-4757-2201-7

R. Aslaner, A. \& Ihsan Boran. (2009). On the geometry of null curves in the Minkowski 4-space. Turk J Math, 33, 265-272. https://doi.org/10.3906/mat-0711-31

R. Lopez. (2008). Differential geometry of curves and surfaces in Lorentz-Minkowski space, Instituto de Matematica Estatistica, University of Sao Paulo, Brazil. 


\section{Copyrights}

Copyright for this article is retained by the author(s), with first publication rights granted to the journal.

This is an open-access article distributed under the terms and conditions of the Creative Commons Attribution license (http://creativecommons.org/licenses/by/3.0/). 